\title{
A comprehensive candidate gene study on bronchial asthma and juvenile idiopathic arthritis
}

\author{
K. Schubert, H. von Bonnsdorf, M. Burke, I. Ahlert, S. Braun, R. Berner, K.A. Deichmann and \\ A. Heinzmann* \\ University Children's Hospital, University of Freiburg, Mathildenstr. 1, 79106 Freiburg, Germany
}

\begin{abstract}
Bronchial asthma and juvenile idiopathic arthritis (JIA) are complex genetic diseases. As both represent chronic inflammatory diseases it is likely that they are at least partially influenced by the same genetic variants. One goal in dissecting the genetics of complex diseases is to identify a genetic risk profile. Therefore it is necessary to genotype polymorphisms in many different pathways. Thus we investigated 48 polymorphisms in 24 genes for association with asthma and/or JIA.

Genotpying was performed on 231 asthmatic children, 86 children with JIA and 270 controls. Association analysis was performed by the Armitage's trend test. Furthermore haplotypes were calculated by FAMHAP.

We found association of polymorphisms within IL-4, CTLA4 and TNFalpha with asthma and/or JIA. Furthermore, the polymorphisms showed an inverse distribution between children with asthma and JIA. However, we were not able to confirm association of most of the previously described candidate genes.

We conclude from our data that it might be very difficult to identify genetic risk profiles for the development of asthma and/or JIA that would be valid across different populations. However, this study adds further evidence that the common genetic background of asthma and JIA is mainly based on polymorphisms in important TH1 and TH2 cytokines.
\end{abstract}

Keywords: Arthritis, asthma, complex disease, polymorphism

\section{Introduction}

Bronchial asthma and juvenile idiopathic arthritis (JIA) are chronic inflammatory diseases. They represent complex genetic diseases. The dissection of the genetics of complex diseases is hampered by the following factors:

Complex diseases are not only caused by a variety of polymorphisms in different genes, but also influenced by the interactions of these variants with each other (epistasis). This is still very difficult to detect by current statistical methods [1]. Besides, environmental factors can provoke the disease even in absence of any

${ }^{*}$ Corresponding author: Dr. Andrea Heinzmann, University Children's Hospital, University of Freiburg, Mathildenstr. 1, 79106 Freiburg, Germany. Tel.: +49 761 2704300; Fax: +49 761 2706372; E-mail: heinzmann@kikli.ukl.uni-freiburg.de. disease-causing polymorphisms (phenocopy); individuals which harbour some genetic risk factors to develop the disease can be completely healthy (incomplete penetrance). Finally, specific environmental factors can interact with genetic variants [2,3].

Despite the difficulty in defining the genetic basis of complex diseases, some progress has been made recently and many candidate genes have been described. The aim behind these studies is to gain new insights into the pathophysiology of the diseases. This should lead to the development of new drugs which act more specific and effective. Including data about the response to a therapy and observed side effects in genetic studies will also enable us to predict which patient might benefit from a special drug [4]. Maybe the most ambitious aim is to identify a genetic risk profile for the development of complex diseases. This would lead to the future scenario where we can perform a genetic test 
soon after birth and calculate the risk for the newborn to develop a complex disease. In case of a genetically increased risk it would be feasible to perform preventive measures very early in life when the organism is most susceptible for such approaches. In order to develop risk profiles for complex diseases it is necessary to genotype polymorphisms within a variety of genes on one clearly defined population.

We are mainly interested in the genetics of bronchial asthma. As several studies have shown that chronic inflammatory diseases share at least some common genetic determinants [5] we also recruited a population of children with JIA. Recently, a comprehensive review on asthma genetics has described 64 genes found at least once in association with asthma or atopy; about 30 of those genes were confirmed in a second population [6]. In this study we mainly focused on those genes which have been found repetitively in association with bronchial asthma until spring 2004. Thus we typed 48 polymorphisms in 24 genes involved in different inflammatory pathways in asthmatic children, children with JIA and controls.

\section{Materials and methods}

\subsection{Asthmatic population}

The asthmatic population has been described in detail before [7]. Briefly, three hundred and twenty one children with suspected asthma (aged 5 to 18 years) were recruited from the south-western part of Germany between July 2000 and January 2003. The probands were characterised at the University Children's Hospital, Freiburg, Germany, using a standardised clinical protocol. An extended medical history was recorded including occurrence and duration of wheezing symptoms, previous and acute medications, severity of previous asthma attacks, previous allergic rhinitis or conjunctivitis, atopic dermatitis and any family history of allergic diseases.

\subsection{Pulmonary function tests}

Pulmonary function tests were performed using standard protocols. In addition exercise induced asthma was diagnosed by subjecting the probands to physical exercise for 6 minutes under standardised conditions. The first spirometry and peak flow measurement were performed after 2-3 minutes and the second after 56 minutes. After 10 more minutes the children inhaled with salbutamol and a third spirometry and peak flow measurement were taken.

To test for bronchial hyperresponsiveness inhalations with increasing, doubling concentrations of histamine (from $0.125 \mathrm{mg} / \mathrm{dl}$ to $8 \mathrm{mg} / \mathrm{dl}$ ) were performed. Testing was stopped after a $15 \%$ fall in FEV1.

\subsection{Asthma definition}

Two hundred and thirty one out of the 321 recruited children were diagnosed as positive for bronchial asthma. The diagnosis was based on a clear-cut history of asthmatic symptoms, the use of anti-asthmatic medication and the existence of bronchial hyperreactivity (BHR). BHR was defined as a fall in FEV1 by at least $15 \%$ in histamine testing or exercise provocation.

\subsection{Population of children with juvenile idiopathic arthritis}

The forms of JIA have been classified by the International League of Associations for Rheumatology [8]. The present study is focussed on patients fulfilling the clinical criteria of chronic arthritis which refers to arthritis of at least 6 weeks duration of unknown cause occurring in children less than 16 years old. In addition, all patients included in the study were tested positive for antinuclear antibodies (ANA) in serum, i.e. titer $>1: 80$. Eighty six children fulfilling this criteria were recruited. One child of this group had asthma but did not show elevated IgE levels.

\subsection{Control population}

Two hundred and seventy randomly chosen probands were used as controls (aged 19 to 40 years). They originate from the same area in the south-western part of Germany. No medical history was taken and no medical testing was performed on controls.

\subsection{Sequencing}

For all polymorphisms three controls (homozygous wildtype, heterozygous and homozygous mutation) were sequenced by the dideoxy chain termination method [9] using the Big Dye Terminator cycle sequencing kit on an ABI 310 sequencer (Applied Biosystems). All the following studies included these three reference individuals for each polymorphism. 


\subsection{Genotyping}

All polymorphisms were genotyped by means of Restriction Fragment Length Polymorphisms. The fragments were resolved on $2 \%$ agarose gels.

\subsection{Statistical analysis}

Association analysis was performed for each polymorphism using the Armitage's trend test. This test takes into account the individuals' genotypes rather than just the alleles, following the guidelines given by Sasieni [10] as implemented in the program FINETTI (Thomas F. Wienker, unpublished data; http://ihg.gsf.de/cgi-bin/hw/hwa1.pl and http://ihg.gsf.de/linkage/download/finetti.zip). Analyses were performed for the asthmatic versus the control and the JIA population and for the JIA versus the control population. Armitage's trend test based on the genotypes remains valid even if HWE does not hold and thus should give meore reliable results [11]. Still, for each of the 48 polymorphisms, we tested separately whether the genotype distribution in the three populations was in HWE, using the program FINETTI.

In addition to the analyses based on single polymorphisms, we performed haplotype frequency estimations for those genes in which at least three polymorphisms were typed. This was done by using the programs FASTEHPLUS [12] and FAMHAP [13].

\subsection{Approval}

The collection of DNA material and the experimental procedures were approved by the Ethical Committee of the University of Freiburg. A statement of informed consent was signed by all participants or in the case of children signed by their parents.

\section{Results}

\subsection{Genotyping}

The polymorphisms under investigation are summarised in Table 1. Besides information about the nature of the polymorphisms, the location, accession number and when applicable amino acid exchange the allelic frequency in the three populations is given.

All but four polymorphisms were in Hardy-WeinbergEuqilibrium in all three populations as calculated by De Finetti (data not shown). The four exceptions were in the control population: $\mathrm{rs} 1800796$ in $I L-6$ ( $p=$ $0.0048), \mathrm{rs} 5743836$ in TLR9 $(p=0.0374), \mathrm{rs} 2243250$ in $I L-4(p=0.0275)$ and rs1800888 in ADRB2 ( $p=$ $0.0387)$. The polymorphisms in $I L-6$ and $A D R B 2$ were very rare and thus the deviation is most likely caused by chance. In addition by testing 48 polymorphisms in three populations a $\mathrm{p}$-value of $<0.05$ is expected by chance seven times.

\subsection{Association analysis}

Pairwise association analyses were performed for asthma - controls, JIA - controls and JIA - asthma by the Armitage's trend test. Significant results are shown in Table 2. The listed p-values have not been corrected for multiple testing.

$I L-4$ was weakly associated with asthma ( $p=$ $0.0451)$. There was no association with the diagnosis JIA per se, however the allelic frequency was different between asthmatics and children with JIA by the p-value of 0.001 .

Polymorphisms within CTLA4 and TNFalpha were not associated with the diseases per se, but again children with JIA differ significantly from children with asthma ( $p=0.030$ and 0.034 respectively).

\subsection{Haplotype analysis}

Haplotypes were calculated for the genes in which at least three polymorphisms were genotyped: $A D A M 33$, CSF2, FCERIB and TLR4. There was no significant difference in haplotype distribution between the populations for any of those genes (data not shown).

\section{Discussion}

In this study we included 48 polymorphisms within 24 candidate genes for bronchial asthma. Most of these genes play an important role in the regulation of immune response in general, thus we investigated the same polymorphisms in a population of another chronic inflammatory disease, that is children with JIA. Despite the fact that most of these polymorphisms have been found repetitively in association with asthma and/or rheumatoid arthritis, we only found association with three genes:

The strongest association was found with the promotor polymorphism -C590T in IL-4. The allelic frequency differed by the p-value of 0.001 between asthmatics and children with JIA. Furthermore the same 
Table 1

Listed are the genes, polymorphisms and their allelic frequencies in children with asthma, controls and children with juvenile idiopathic arthritis

\begin{tabular}{|c|c|c|c|c|c|c|c|}
\hline Gene & Polymorphism & Location & AA exchange & rs number & Frequency Asthma & Controls & JIA \\
\hline \multirow{4}{*}{ ADAM33 } & $\mathrm{C} / \mathrm{G}$ & Exon 19 & & rs 528557 & 0.299 & 0.257 & 0.274 \\
\hline & $\mathrm{A} / \mathrm{G}$ & Intron 19 & & rs597980 & 0.421 & 0.395 & 0.418 \\
\hline & $\mathrm{G} / \mathrm{T}$ & Intron 19 & & rs44707 & 0.419 & 0.393 & 0.429 \\
\hline & $\mathrm{A} / \mathrm{G}$ & Exon 20 & M764T & rs2280091 & 0.156 & 0.120 & 0.149 \\
\hline \multirow[t]{2}{*}{ ADRB2 } & $\mathrm{A} / \mathrm{G}$ & Exon 1 & $\mathrm{R} 16 \mathrm{G}$ & rs 1042713 & 0.380 & 0.379 & 0.429 \\
\hline & $\mathrm{C} / \mathrm{T}$ & Exon 1 & T164I & rs 1800888 & 0.009 & 0.013 & 0.006 \\
\hline \multirow[t]{2}{*}{ CARD15 } & $\mathrm{C} / \mathrm{T}$ & Exon 4 & R684W & rs5743276 & 0.070 & 0.065 & 0.094 \\
\hline & $3020 \mathrm{iC}$ & Intron 11 & & & 0.041 & 0.030 & 0.029 \\
\hline \multirow[t]{2}{*}{ CCR3 } & $\mathrm{C} / \mathrm{T}$ & Intron 2 & & rs1491962 & 0.420 & 0.421 & 0.367 \\
\hline & $\mathrm{C} / \mathrm{T}$ & Exon 3 & & rs 4987053 & 0.074 & 0.069 & 0.088 \\
\hline CCR5 & $\mathrm{Wt} / \Delta 580-611$ & Exon 1 & & rs333 & 0.094 & 0.116 & 0.071 \\
\hline \multirow[t]{3}{*}{$\mathrm{CSF} 2$} & $\mathrm{C} / \mathrm{T}$ & Intron 3 & & rs 25881 & 0.180 & 0.169 & 0.165 \\
\hline & $\mathrm{T} / \mathrm{C}$ & Exon 4 & I117T & rs 25882 & 0.221 & 0.213 & 0.200 \\
\hline & G/A & Promotor -1440 & & rs2069616 & 0.424 & 0.429 & 0.382 \\
\hline \multirow[t]{2}{*}{ CTLA4 } & $\mathrm{C} / \mathrm{T}$ & Promotor -318 & & rs5742909 & 0.116 & 0.111 & 0.061 \\
\hline & $\mathrm{A} / \mathrm{G}$ & Exon 1 & T17A & rs 231775 & 0.350 & 0.377 & 0.410 \\
\hline \multirow[t]{3}{*}{ FCER1B } & $\mathrm{A} / \mathrm{G}$ & Intron 2 & & rs 2583476 & 0.413 & 0.399 & 0.428 \\
\hline & $\mathrm{A} / \mathrm{G}$ & Exon 7 & E237G & rs569108 & 0.029 & 0.024 & 0.011 \\
\hline & $\mathrm{C} / \mathrm{T}$ & Exon 7 & & rs 2847655 & 0.419 & 0.401 & 0.429 \\
\hline \multirow[t]{2}{*}{ IL-2 } & $\mathrm{G} / \mathrm{T}$ & Exon 1 & L38R & rs2069763 & 0.397 & 0.347 & 0.369 \\
\hline & $\mathrm{T} / \mathrm{G}$ & Promotor -385 & & rs2069782 & 0.267 & 0.312 & 0.259 \\
\hline \multirow[t]{2}{*}{ IL-3 } & $\mathrm{T} / \mathrm{C}$ & Promotor -68 & & rs31480 & 0.213 & 0.216 & 0.226 \\
\hline & $\mathrm{C} / \mathrm{T}$ & Exon 1 & $\mathrm{P} 27 \mathrm{~S}$ & rs40401 & 0.222 & 0.222 & 0.238 \\
\hline IL-4 & $\mathrm{C} / \mathrm{T}$ & Promotor -590 & & rs 2243250 & 0.214 & 0.163 & 0.110 \\
\hline \multirow[t]{2}{*}{ IL-6 } & $\mathrm{G} / \mathrm{C}$ & Promotor -572 & & rs 1800796 & 0.061 & 0.057 & 0.050 \\
\hline & $\mathrm{G} / \mathrm{C}$ & Promotor -174 & & rs1800795 & 0.394 & 0.403 & 0.429 \\
\hline \multirow[t]{2}{*}{ IL-10 } & $\mathrm{C} / \mathrm{A}$ & Promotor -571 & & rs1800872 & 0.248 & 0.267 & 0.247 \\
\hline & $\mathrm{A} / \mathrm{G}$ & Exon 5 & & rs3024498 & 0.228 & 0.235 & 0.212 \\
\hline MCP1 & G/A & Promotor -2518 & & rs1024611 & 0.266 & 0.275 & 0.225 \\
\hline \multirow[t]{2}{*}{ MIF } & $\mathrm{C} / \mathrm{G}$ & Intron 2 & & rs2070766 & 0.161 & 0.142 & 0.205 \\
\hline & $\mathrm{C} / \mathrm{G}$ & Promotor -270 & & rs755622 & 0.177 & 0.151 & 0.212 \\
\hline NOS1 & $\mathrm{C} / \mathrm{G}$ & Exon 15 & & rs13391 & 0.305 & 0.307 & 0.260 \\
\hline NOS3 & $\mathrm{G} / \mathrm{T}$ & Exon 7 & E298D & rs1799983 & 0.323 & 0.299 & 0.304 \\
\hline \multirow[t]{2}{*}{ PAFAH } & $\mathrm{A} / \mathrm{G}$ & Exon 8 & I198T & rs 1805018 & 0.046 & 0.041 & 0.027 \\
\hline & $\mathrm{A} / \mathrm{G}$ & Exon 12 & V370A & rs1051931 & 0.243 & 0.225 & 0.202 \\
\hline \multirow[t]{2}{*}{ RANTES } & $\mathrm{A} / \mathrm{G}$ & Promotor -177 & & rs1800825 & 0.022 & 0.028 & 0.041 \\
\hline & $\mathrm{C} / \mathrm{T}$ & Promotor -471 & & rs2107538 & 0.216 & 0.195 & 0.185 \\
\hline \multirow[t]{2}{*}{ SELP } & $\mathrm{G} / \mathrm{A}$ & Exon 7 & S330N & rs6131 & 0.209 & 0.213 & 0.218 \\
\hline & $\mathrm{G} / \mathrm{T}$ & Exon 12 & V640L & rs6133 & 0.098 & 0.110 & 0.065 \\
\hline \multirow[t]{2}{*}{ TNFalpha } & $\mathrm{C} / \mathrm{T}$ & Promotor -1037 & & rs1799724 & 0.110 & 0.147 & 0.170 \\
\hline & G/A & Promotor -308 & & rs 1800629 & 0.167 & 0.146 & 0.145 \\
\hline TNFbeta & $\mathrm{A} / \mathrm{G}$ & Intron 1 & & rs909253 & 0.341 & 0.323 & 0.286 \\
\hline \multirow[t]{4}{*}{ TLR4 } & $\mathrm{A} / \mathrm{G}$ & Promotor -6142 & & & 0.308 & 0.301 & 0.312 \\
\hline & $\mathrm{T} / \mathrm{C}$ & Promotor -5723 & & & 0.107 & 0.119 & 0.129 \\
\hline & $\mathrm{A} / \mathrm{G}$ & Exon 2 & D259G & rs4986790 & 0.041 & 0.048 & 0.041 \\
\hline & $\mathrm{C} / \mathrm{T}$ & Exon 2 & T359I & rs4986791 & 0.048 & 0.045 & 0.041 \\
\hline \multirow[t]{2}{*}{ TLR9 } & $\mathrm{A} / \mathrm{G}$ & Promotor -1237 & & rs5743836 & 0.129 & 0.150 & 0.100 \\
\hline & $\mathrm{C} / \mathrm{T}$ & Exon 2 & & rs 352140 & 0.434 & 0.455 & 0.488 \\
\hline
\end{tabular}

Abbreviations: ADAM33: A disintegrin and metalloproteinase domain 33; ADBR2: $\beta 2$-adrenergic receptor; CARD15: Caspase recruitment domain-containing protein 15; CCR: Chemokin receptor; CSF2: colony stimulating factor 2; CTLA4: Cytotoxic T lymphocyte-associated 4; FCER1B: Fc $\varepsilon$ receptor $\beta$ chain (high affinity IgE receptor); IL: Interleukin; MCP1: monocyte chemotactic protein; MIF: macrophage migration inhibitory factor; NOS: nitric oxide synthase; PAFAH: platelet-activating factor acetylhydrolase; RANTES: regulated upon activation, normally T-expressed, and presumably secreted; SELP: selectin P; TNF: tumor necrosis factor; TLR: Toll like recptor.

polymorphism was weakly associated with bronchial asthma. These results resemble the one we obtained with a $I L-13$ variant in the same populations [14]. IL4 and IL-13 represent the strongest TH2 cytokines. According to the classical TH1-TH2 paradigm they promote TH2 diseases like bronchial asthma and at the same time suppress TH1 diseases like rheumatoid arthritis. Thus the results are in support for this TH1TH2 balance theory.

In addition we found association of polymorphisms 
Table 2

Results of the association study by the Armitage's trend test. Listed are only the polymorphisms with significant association

\begin{tabular}{lcccc}
\hline Gene & Polymorphism & Asthma-controls & JIA-controls & Asthma-JIA \\
\hline CTLA4 & rs5742909 & 0.451 & 0.086 & 0.030 \\
IL-4 & rs 2243250 & 0.045 & 0.088 & 0.001 \\
TNFalpha & rs1799724 & 0.083 & 0.434 & 0.034 \\
\hline
\end{tabular}

within CTLA4 and TNFalpha by comparing asthmatic children with children suffering of JIA. However both assocations are quite weak $(p=0.030$ and 0.034 , respectively) and might be reached by chance. Association of CTLA4 with asthma has been described before [15], and an inverse association with arthritis is also known [16]. This would be in favour of the TH1TH2 balance of the immune system, too.

Our data are in contrast to several previously published studies. This could be due to several reasons: The size of the study population is essential. Using a small sample size can result in false negative results. The risk of missing true association is especially high in complex diseases in which the effect of one single polymorphism on the disease is quite small [17]. Our asthmatic population might be of a reasonable size to identify major genes for asthma (the power for detecting association ranges from 0.923 to 0.967 for polymorphisms with an allelic frequency of 0.2 to 0.5 ). However, the JIA population is quite small and thus association could be missed (statistical power from 0.689 to 0.780 for polymorphisms with an allelic frequency of 0.2 to 0.5 ). In addition, we typed in average two polymorphisms in each gene and thus the real-disease causing variant could be missed. The power of association analyses can be improved by including haplotype analyses. We have shown in a previous study within the same asthmatic population that five single polymorphisms in $I L-15$ are not associated with asthma whereas haplotypes built up out of these polymorphisms showed highly significant association [18]. In the here presented study we also calculated haplotypes for all genes in which at least three polymorphisms were genotyped. However, we did not find any significant result.

An emerging new field in asthma genetics are the potential interactions between genes and environment [2]. Not including these data into analyses might therefore lead to false negative results. We have no data about environmental factors in our study populations so we can not exclude the possibility that we missed such an association in our sample.

Another important factor in association studies is the characterisation of controls. We used randomly chosen controls, which have not been characterised for any phenotype. Thus it is highly likely that we have included some individuals with asthma or JIA in the control population. This will enhance the possibility of a type 2 error.

Finally, distortion of association results is simply produced by the fact that positive association studies are far more easily published than negative results [6]. Therefore the impact of a gene on the development of a disease might be over estimated.

To give a complete picture of the genetics of asthma and JIA in our populations it should be mentioned that in previous published studies we did not find association of polymorphisms within $I L-18$, matrix metallo proteinase 9, UGRP1, CD14 and TGF-beta with the diseases. In contrast, we did find association of bronchial asthma with $I L-8, I L-13$ and $I L-15$ and of JIA with $I L-13$ and $I L-15$.

It is striking that until now we have only found association of asthma or JIA per se with different Interleukins though we have investigated a wide range of genes participating in different pathways. It seems unlikely that this is due to a special genetic background or environment in Germany as other studies in German children have also identified other candidate genes [19, 20].

When comparing the genetics of JIA and bronchial asthma the results might suggest the following: On the one hand the common genetic background of both diseases is influenced by the opposite alleles of polymorphisms in strong TH1 and TH2 cytokines and thus in accordance to the TH1/TH2 balance hypothesis. On the other hand polymorphisms in genes coding for proinflammatory proteins show similar distribution between both populations and thus might have the same effect on both diseases. In this regard the classical TH1-TH2 paradigm is still too simple for the explanation of the underlying immune response in chronic inflammatory disease. Recent studies are now focussing on the so called regulatory T-cells [21].

However, we have to conclude from our data that it will be very difficult to identify a genetic risk profile for the development of asthma or JIA which will be valid across different study populations - even in the same country. 


\section{Acknowledgements}

This project was supported by a grant from the Deutsche Forschungsgemeinschaft (DFG He 386/4-2). The collection of asthmatic children has partly been supported by a grant from the NIH (NIH R01 HL66533$01)$.

\section{References}

[1] J.N. Hirschhorn and M.J. Daly, Genome-wide association studies for common diseases and complex traits, Nat Rev Genet. 6(2) (2005), 95-108.

[2] M. Kabesch, C. Hoefler, D. Carr, W. Leupold, S.K. Weiland and E. von Mutius, Glutathione S transferase deficiency and passive smoking increase childhood asthma, Thorax. 59(7) (2004), 569-573.

[3] W. Eder, W. Klimecki, L. Yu, E. von Mutius, J. Riedler, C. Braun-Fahrlander et al., Toll-like receptor 2 as a major gene for asthma in children of European farmers, J Allergy Clin Immunol. 113(3) (2004), 482-488.

[4] G. Ferraccioli, M. De Santis and B. Tolusso, Pharmacogenetics/pharmacogenomics and antirheumatic drugs in rheumatology, Pharmacogenomics. 5(8) (2004), 1107-1116.

[5] K.G. Becker, R.M. Simon, J.E. Bailey-Wilson, B. Freidlin, W.E. Biddison, H.F. McFarland et al., Clustering of non-major histocompatibility complex susceptibility candidate loci in human autoimmune diseases, Proc Natl Acad Sci USA 95(17) (1998), 9979-9984.

[6] S. Hoffjan, D. Nicolae and C. Ober, Association studies for asthma and atopic diseases: a comprehensive review of the literature, Respir Res. 4(1) (2003), 14.

[7] A. Heinzmann, I. Ahlert, T. Kurz, R. Berner and K.A. Deichmann, Association study suggests opposite effects of polymorphisms within IL8 on bronchial asthma and respiratory syncytial virus bronchiolitis, J All Clin Immunol 114(3) (2004), 671-676.

[8] R.E. Petty, T.R. Southwood, J. Baum, E. Bhettay, D.N. Glass, P. Manners et al., Revision of the proposed classification criteria for juvenile idiopathic arthritis: Durban, 1997, J Rheumatol 25 (1998), 1991-1994.

[9] F. Sanger, S. Nicklen and A.R. Coulson, DNA sequencing with chain-terminating inhibitors. 1977, Biotechnology 24 (1992), 104-108.
[10] P.D. Sasieni, From genotypes to genes: doubling the sample size, Biometrics 53(4) (1997), 1253-1261.

[11] J. Xu, A. Turner, J. Little, E.R. Bleecker and D.A. Meyers, Positive results in association studies are associated with departure from Hardy-Weinberg equilibrium: hint for genotyping error? Hum Genet 111(6) (2002), 573-574.

[12] J.H. Zhao and P.C. Sham, Faster haplotype frequency estimation using unrelated subjects, Hum Hered 53(1) (2002), 36-41.

[13] T. Becker and M. Knapp, Maximum-likelihood estimation of haplotype frequencies in nuclear families, Genet Epidemiol. 27(1) (2004), 21-32.

[14] A. Heinzmann, S.P. Jerkic, K. Ganter, T. Kurz, S. Blattmann, L. Schuchmann et al., Association study of the IL13 variant Arg110Gln in atopic diseases and juvenile idiopathic arthritis, J Allergy Clin Immunol. 112(4) (2003), 735-739.

[15] T.D. Howard, D.S. Postma, G.A. Hawkins, G.H. Koppelman, S.L. Zheng, A.K. Wysong et al., Fine mapping of an IgEcontrolling gene on chromosome 2q: Analysis of CTLA4 and CD28, J Allergy Clin Immunol. 110(5) (2002), 743-751.

[16] M.R. Rodriguez, A. Nunez-Roldan, F. Aguilar, A. Valenzuela, A. Garcia and M.F. Gonzalez-Escribano, Association of the CTLA4 3' untranslated region polymorphism with the susceptibility to rheumatoid arthritis, Hum Immunol. 63(1) (2002), 76-81.

[17] K.E. Lohmueller, C.L. Pearce, M. Pike, E.S. Lander and J.N. Hirschhorn, Meta-analysis of genetic association studies supports a contribution of common variants to susceptibility to common disease, Nat Genet. 33(2) (2003), 177-182.

[18] T. Kurz, K. Strauch, H. Dietrich, S. Braun, S. Hierl, S.P. Jerkic et al., Multilocus haplotype analyses reveal association between 5 novel IL-15 polymorphisms and asthma, J Allergy Clin Immunol. 113(5) (2004), 896-901.

[19] M. Kabesch, W. Peters, D. Carr, W. Leupold, S.K. Weiland and E. von Mutius, Association between polymorphisms in caspase recruitment domain containing protein 15 and allergy in two German populations, J Allergy Clin Immunol. 111(4) (2003), 813-817.

[20] R.G. Nickel, V. Casolaro, U. Wahn, K. Beyer, K.C. Barnes, B.S. Plunkett et al., Atopic dermatitis is associated with a functional mutation in the promoter of the C-C chemokine RANTES, J. Immunol. 164(3) (2000), 1612-1616.

[21] J.J. Smit, G. Folkerts and F.P. Nijkamp, Mycobacteria, genes and the 'hygiene hypothesis', Curr Opin Allergy Clin Immunol. 4(1) (2004), 57-62. 


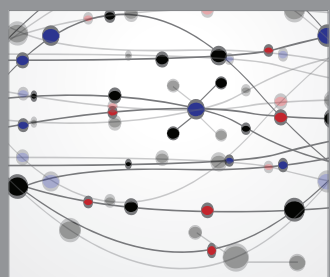

The Scientific World Journal
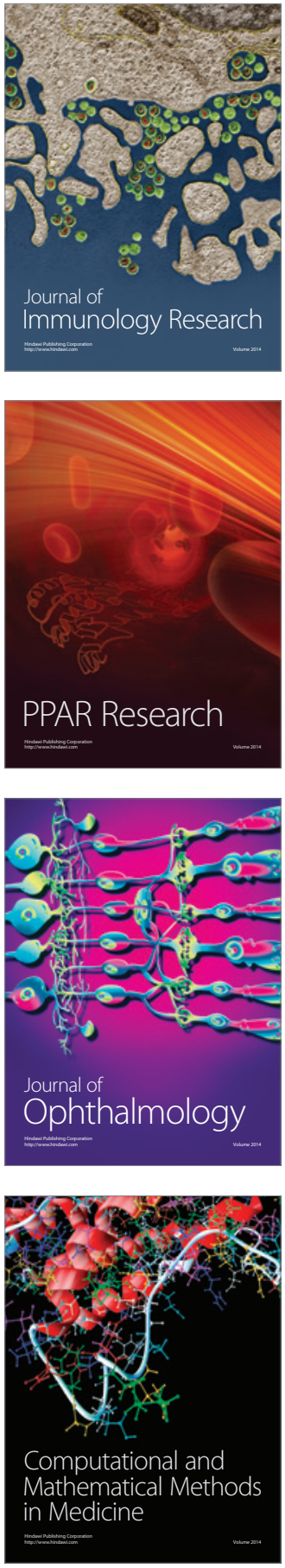

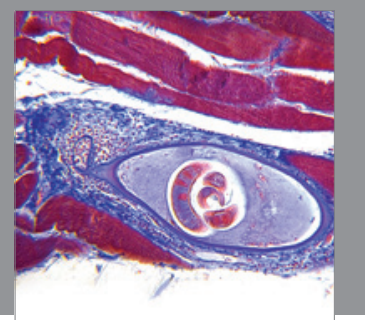

Gastroenterology

Research and Practice
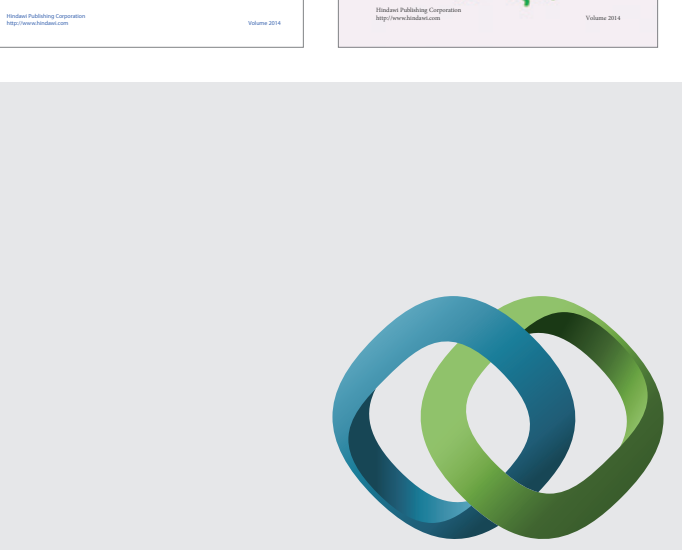

\section{Hindawi}

Submit your manuscripts at

http://www.hindawi.com
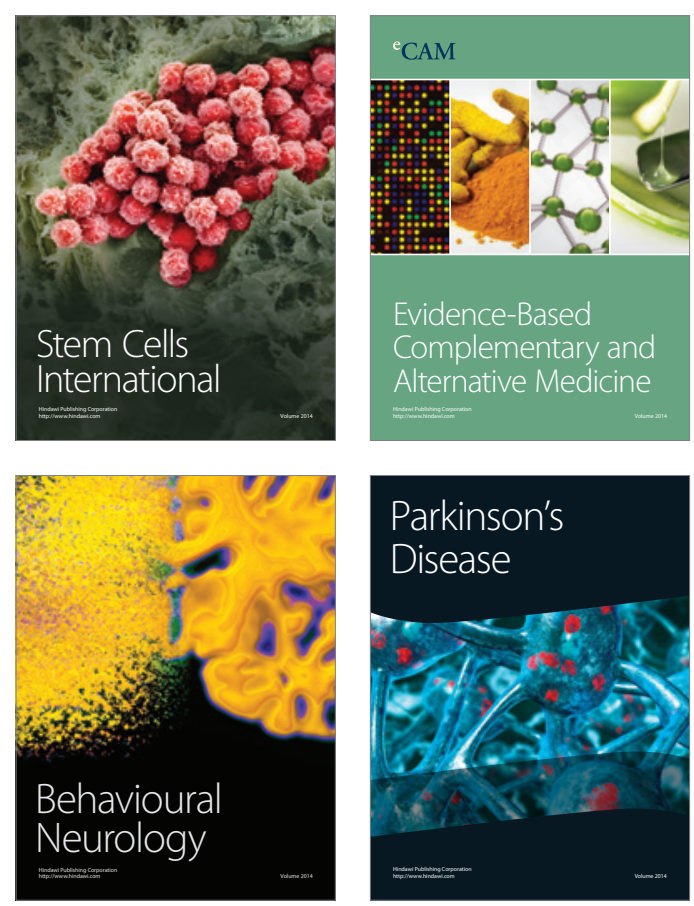

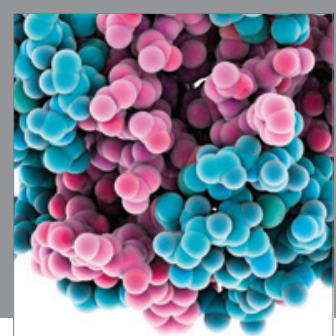

Journal of
Diabetes Research

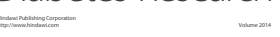

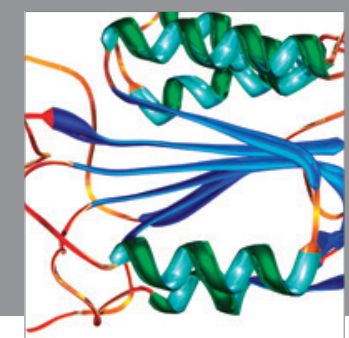

Disease Markers
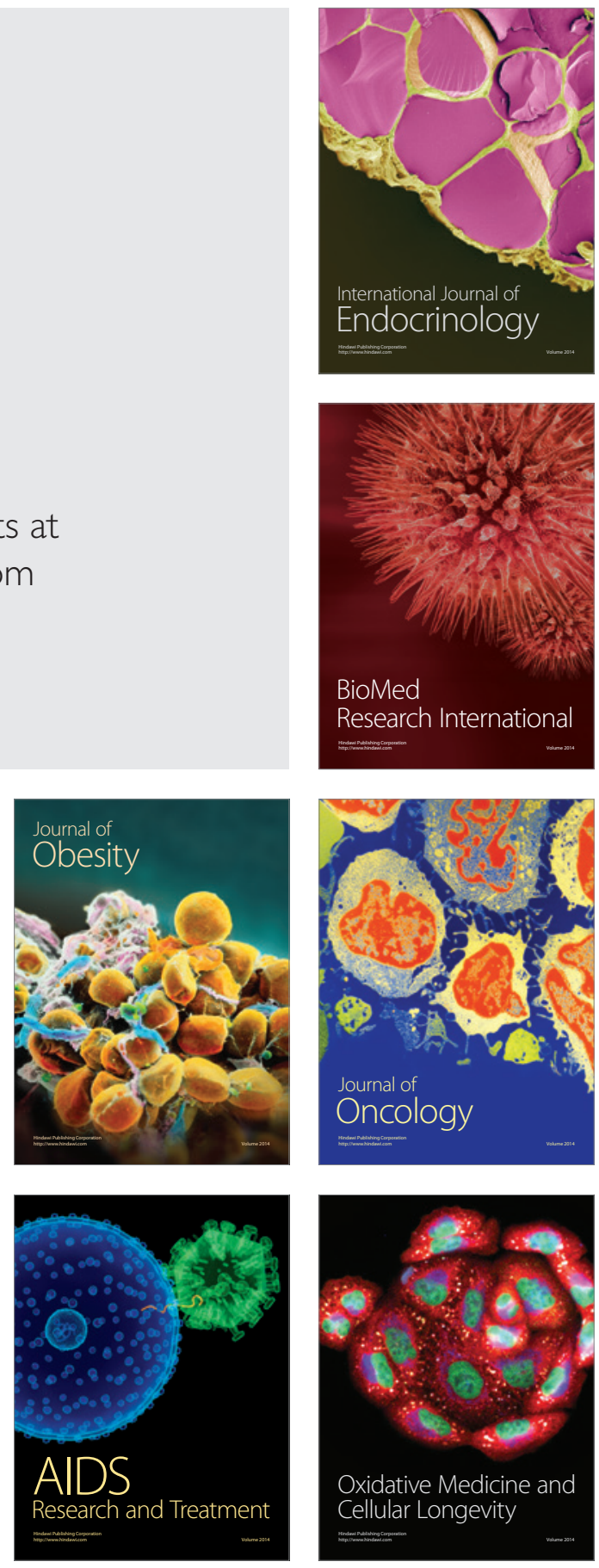